\title{
EDITORIAL
}

\section{NORMAL AND ABNORMAL BINOCULAR VISION}

In this issue of Eye which contains the papers from the Cambridge Symposium on Binocularity the most important problem to be addressed was the origin of infantile strabismus. What is the primary pathogenic factor: a sensory defect in the visual cortex that prevents motor fusion or a motor defect that prevents the development of sensory binocularity? Although neurophysiologists have found many correlates of normal and abnormal binocular interaction that help to understand how the system works, animal experiments have not yet been able to clarify the problem of whether a sensory or a motor defect is the primary abnormality. The developmental studies in infants of families with a disposition for strabismus by Braddick, reported in this issue, seem to indicate that a motor rather than a sensory defect is the primary abnormality.

The practical implications for the management of infantile strabismus can be considerable. If the primary defect is sensory, surgical alignment of the eyes, even achieved very early, would not cure the patient, but if the primary defect is motor, immediate surgical alignment should lead to normal binocularity. It may, however, turn out that both possibilities exist or interact in various proportions so that the chances of a functional cure of infantile strabismus are quite different from family to family, or even from individual to individual.

A possible approach might be to investigate the visual cortex of newborn monkeys with a genetic disposition for strabismus such as those described by Boothe. However, in order to understand the mechanism it is important to understand the underlying neuro-architecture of binocular vision. Casagrande finds that although eye-specific inputs can still be segregated within layer IV of the primary visual cortex, they are combined at the next stage of cortical processing, so that many binocular cells are found in all layers outside of layer IV. There is suggestive evidence that binocular interactions responsible for stereopsis occur already at the level of the lateral geniculate nucleus, but this issue is still unclear, as is the role of the feedback pathway from layer VI of the visual cortex to the lateral geniculate nucleus. Both $\mathrm{M}$ - and $\mathrm{P}$-channels play a role in stereopsis.

Much work has now been completed on primates. The sensitive period for the development of binocularity has been studied in Crawford's model using vertical prisms. Also, the interactions between the motor and sensory pathways are well illustrated in Judge's studies of vergence and accommodation. Although most of the supranuclear neurons that discharge proportional to vergence also discharge with accommodation, there are a few neurons that differentiate between these two functions. The reason why only some hypermetropic infants develop an accommodative esotropia could be that those who develop strabismus may have an inflexible link between accommodation and vergence. Judge rightly emphasises that it is not the ratio of accommodation to vergence that needs to be adjusted to make accommodative vergence appropriate in hypermetropia, but the level of tonic vergence. The existence of proximal vergence is highlighted by the fact that the implied depth influences the near triad when subjects view a painting with strong perspective clues.

If monkeys are made esotropic for a certain period during infancy by muscle surgery they can recover motor fusion, even though they retain deficiencies in sensory fusion. Harwerth points out that this experimental situation is similar to the monofixation syndrome and to microtropia, so that these clinical entities, usually thought to be due to a primary sensory abnormality, may in fact be the consequence of a transitory motor failure.

Guillery reviews why albinos and other hypopigmented mutants lack normal binocular vision. The main abnormality shared by these genetically different individuals is that fibres originating from the temporal retina cross in the chiasm. The mechanism that causes this misrouting is not understood; nor is maldevelopment of the fovea typically encountered in these subjects. As a consequence of the chiasmatic misrouting there are also abnormalities of the central 
visual relays, including corticofugal projections. This latter finding may explain the nystagmus that albino patients usually have.

In monkeys with an artificial strabismus the interaction of inputs from the two eyes can be studied without making the stimuli different. As Sengpiel reports, most cells in V1 respond with suppression as soon as the squinting eye is exposed to a similar (iso-oriented) grating as the dominant eye.

Suppression is one of the mechanisms that prevents double vision in the case of strabismus, the other being abnormal retinal correspondence. Harrad points out that, to measure suppression, the stimuli applied to each eye have to be different. Unfortunately the difference between the two images alters the natural viewing condition and, most likely, exaggerates suppression. Harrad makes an analogy here with Heisenberg's Uncertainty Principle, according to which a physical event is modified if investigated. To circumvent this problem Mehdorn (Doc Ophthalmol 1989;71:1-18) applied stereo perimetry in microstrabismic patients who had a suppression scotoma at the fixation point in the deviating eye on conventional testing (Bagolini's striated glasses). He found that stereo acuity is best near the fixation point (although certainly not normal). This would be impossible if a suppression scotoma were present under the quasi-natural stereo viewing conditions. Mehdorn's data suggest that anomalous retinal correspondence is more, and suppression less, important than usually considered in the prevention of double vision in strabismus.

In large angle strabismus the fovea of the deviating eye provides a second area of high resolution in the binocular visual field which can, according to Herzau's observations, be irritating, although the egocentric and relative localisation of the deviating eye can be correct, thanks to a harmonious anomalous retinal correspondence. After squint surgery the angle of correspondence can change by adaptation, but a change to normal binocularity with stable interocular connections cannot be achieved.

Hess relates the monocular psychophysical deficits to the stereo deficits in amblyopic patients. Amblyopia consists of three main parts: acuity loss, contrast sensitivity loss and spatial uncertainty. The acuity loss interferes with the fine (Fourier, linear), not the coarse (non-Fourier, non-linear) stereo mechanism. Only severe deficits in contrast sensitivity affect stereo computation, more so the fine than the coarse mechanism. The positional uncertainty of amblyopic eyes seems to be the main impediment for stereo mechanisms because it interferes with a proper matching of contours presented to the two eyes (see also Parker's article).

In a prospective study by Atkinson in which hypermetropic children $(\geqslant 2.5 \mathrm{D})$ were treated by wearing a spectacle correction (with a $1 \mathrm{D}$ deduction) or not treated, showed a statistically significant difference suggesting that correction of a hypermetropia of $\geqslant 2.5 \mathrm{D}$ is a prophylactic measure against strabismus. Using partial correction, emmetropisation was not hindered (whether full correction would have done so is not known). The results are also of theoretical interest since they support the view that a failure of motor alignment, rather than a primary sensory defect, is often the cause of infantile strabismus. Atkinson and her group have another study in progress in which the predictive value of non-cycloplegic determination of the accommodative lag at a distance of $0.75 \mathrm{~m}$ is tested. This second study promises to be of great practical relevance.

Another difficult problem is the relationship between infantile strabismus and latent nystagmus. Kommerell has a hypothesis which is at variance with the idea that latent nystagmus might constitute a tonic drive which leads to convergent strabismus. He suggests that at first the binocularity in the visual cortex is impaired, either as a primary defect or as a consequence of misalignment of the eyes. Secondly, the reduced binocularity prevents maturation of signal transmission from the visual cortex to the brain stem such that slip control of the retinal image remains maldeveloped, particularly for temporally directed optokinetic stimuli. Lastly the optokinetic asymmetry which becomes evident in latent nystagmus reflects a tonic conjugate preponderance, directed nasally with reference to the fixating eye. A cortical asymmetry tapped by motion VEP (Norcia) may not suffice to explain the optokinetic asymmetry.

The unique atmosphere of the Cambridge Symposium organised by Mr Peter Watson for the past 25 years creates a fascination which passes into all those topics which have been discussed in depth. The fascination of this particular topic which updates our concepts of binocularity, is, as Braddick has pointed out, of particular importance to neurophysiological research because information coming independently from both eyes has to be co-ordinated in the brain. The study of binocular vision can thus lead the way to understanding the broader issues of brain development and organisation, especially the ways through which the brain adapts to, and correlates, information derived from many sources and presented simultaneously.

Guntram Kommerell 